\title{
Seroepidemiology of Toxocara Infection in Patients With Vision Impairment and Blindness in Durango, Mexico
}

\author{
Cosme Alvarado-Esquivel ${ }^{\mathrm{a}, \mathrm{c}}$, Jesus Hernandez-Tinoco ${ }^{\mathrm{b}}$, Luis Francisco Sanchez-Anguiano ${ }^{\mathrm{b}}$
}

\begin{abstract}
Background: Toxocara infection is a cause of vision impairment and blindness. We aimed to determine the seroprevalence and correlates of Toxocara infection in patients suffering from vision impairment and blindness in Durango City, Mexico.
\end{abstract}

Methods: Through a cross-sectional seroprevalence study, 204 patients with vision impairment and 19 blind patients were studied for the presence of anti-Toxocara IgG antibodies in Durango City, Mexico. Seroprevalence association with socio-demographic, housing, clinical, and behavioral characteristics of participants was also investigated.

Results: Five (2.5\%) of the 204 patients with vision impairment, and none of the 19 patients with blindness were positive for anti-Toxocara IgG antibodies. In total, five of the 223 (2.2\%) patients were positive for anti-Toxocara IgG antibodies. Seropositivity to Toxocara was not associated with age, sex, educational level, socio-economic status, presence of underlying diseases or behavioral characteristics of the patients. In contrast, multivariate analysis showed that Toxocara seropositivity was associated with living in a house with soil floor (odds ratio $(\mathrm{OR})=11.14 ; 95 \%$ confidence interval $(\mathrm{CI})$ : 1.57 - 78.74; $\mathrm{P}=$ $0.01)$.

Conclusions: This is the first report of Toxocara exposure in patients with vision impairment and blindness in Mexico, and of an association of Toxocara exposure with living in a house with soil floors. Results suggest a low Toxocara exposure in these patients in Durango, Mexico. The risk factor associated with Toxocara exposure identified in this study warrants for further investigation.

Manuscript accepted for publication November 18, 2014

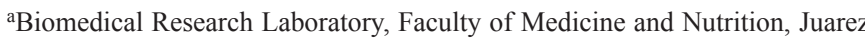
University of Durango State, Avenida Universidad S/N, 34000 Durango, Mexico

'Institute for Scientific Research "Dr. Roberto Rivera-Damm", Juarez University of Durango State, Avenida Universidad S/N, 34000 Durango, Mexico ${ }^{\mathrm{c}}$ Corresponding Author: Cosme Alvarado-Esquivel, Laboratorio de Investigacion Biomedica, Facultad de Medicina y Nutricion, Avenida Universidad S/N, 34000 Durango, Dgo, Mexico. Email: alvaradocosme@yahoo.com

doi: http://dx.doi.org/10.14740/jocmr2032w
Keywords: Case-control study; Epidemiology; Vision impairment; Risk factors; Seroprevalence; Toxocariasis

\section{Introduction}

Toxocara infection is an important cause of vision impairment and blindness [1] and has a worldwide distribution [2-4]. Toxocariasis is a neglected disease caused by several species of Toxocara including canis, cati and other species [5]. There are several routes of Toxocara infection including ingestion of parasite eggs from soil contaminated with excrement shed by dogs and cats infected with Toxocara $[6,7]$, and by ingestion of Toxocara larvae from undercooked giblets $[8,9]$. After infection, Toxocara disseminates in the body by the bloodstream towards eyes, lungs, liver, muscles, and central nervous system $[2,10]$. The clinical spectrum of Toxocara infection varies from asymptomatic to severe disease $[2,3,11]$. Toxocariasis can be limited to affection of eyes and optic nerves [2]; however, permanent blindness may occur $[12,13]$.

Very little is known about the epidemiology of Toxocara infection in patients suffering from eye diseases in Mexico in general and there is lack of knowledge in the northern Mexican state of Durango in particular. In a recent study in Mexico City, researchers found Toxocara seropositivity in eight of 21 patients with ankylosing spondylitis and acute non-granulomatous anterior uveitis [14]. We are not aware of any study aimed to determine the magnitude of Toxocara infection in patients suffering from vision impairment and blindness in Mexico. Therefore, we sought to determine the seroprevalence and correlates of Toxocara infection in patients with vision impairment and blindness in Durango City, Mexico.

\section{Materials and Methods}

\section{Study population}

Through a cross-sectional study using serum samples from a recent Toxoplasma gondii serosurvey [15], we determined the prevalence of anti-Toxocara IgG antibodies in patients suffering from vision impairment or blindness. Inclusion criteria for 
the patients were: 1) patients suffering from vision impairment or blindness attending public settings: the Mexican Social Security Institute (IMSS), Secretary of Health (SS), and State Government (SG); 2) aged 8 years and older; and 3) who accepted to participate in the study. Socio-economic status, sex, and educational level were not restrictive criteria for enrollment. In total, 223 patients were included in the study, 104 of them attended a public ophthalmology center (SG), 100 attended a public hospital for ophthalmology consultations (IMSS), and 19 attended a school for blind people (SG). Patients included 140 females and 83 males aged 8 - 96 years old (mean $50.49 \pm 21.10$ years)

\section{Socio-demographic, housing, clinical and behavioral char- acteristics of patients}

The general characteristics of the patients were obtained with the aid of a standardized questionnaire. Items of socio-demographic data included age, sex, educational level, occupation and socio-economic status. The variable occupation was divided into two subgroups focused on the presence or absence of occupational exposure to soil (gardening or agriculture). We obtained the housing conditions of the patients by using the criteria of Bronfman et al [16]. For this purpose, we obtained information about the crowding at home (number of persons and rooms in the house), type of flooring of the house, availability of drinkable water, and form of elimination of excreta. Clinical data explored included the diagnosis of vision impairment, presence of underlying diseases, history of surgeries, blood transfusions or transplantations, presence of treatment, response to treatment, and presence of other impairments (in memory, reflexes or hearing). Behavioral data included contact with cats and dogs, foreign traveling, consumption of meat (pork, beef, goat, lamb, boar, chicken, turkey, pigeon, duck, rabbit, venison, squirrel, horse, opossum, or other), raw or undercooked meat and dried or processed meat (ham, sausages or chorizo), unwashed raw vegetables and fruits, drinking untreated water or unpasteurized milk, and frequency of eating away from home (in restaurants or fast food outlets).

\section{Laboratory tests}

Sera from participants were kept frozen at $-20{ }^{\circ} \mathrm{C}$ until analyzed. We determined the presence of anti-Toxocara IgG antibodies in the sera with a commercially available enzyme immunoassay "Toxocara" kit (Diagnostic Automation, Inc., Calabasas, CA, USA). The cut off for seropositivity was an absorbance reading equal to or greater than 0.3 optical density units. We included negative and positive controls in each assay and performed the tests following the manufacturer's instructions.

\section{Statistical analysis}

We used the software Epi Info version 7 and SPSS version
15.0 (SPSS Inc., Chicago, IL, USA) to analyze the data. For calculation of the sample size, we used a $95 \%$ confidence level, a reference seroprevalence of $13 \%$ [13] as the expected frequency of exposure in controls, and $5 \%$ of confidence limits. The result of the sample size calculation was 164 patients. The Pearson's Chi-squared test and the two-tailed Fisher's exact test (when values were small) were used to assess the association between Toxocara seropositivity and the socio-demographic, housing, clinical and behavioral characteristics of the patients. The association of Toxocara infection with the characteristics of the patients was further analyzed by multivariate analysis. As a strategy to include variables in the multivariate analysis, we selected variables with a $\mathrm{P}$ value $<0.10$ obtained in the bivariate analysis. Odds ratios (ORs) and 95\% confidence intervals (CIs) were calculated by means of the backward stepwise regression method. We used the Hosmer-Lemeshow goodness of fit test to assess the fitness of our regression model. Statistical significance was set at a $\mathrm{P}$ value less than 0.05 .

\section{Ethical aspects}

In this study, only archival serum samples and questionnaires from a previous survey [15] were used. The Institutional Ethical Committee of the Mexican Institute for Social Security in Durango City, Mexico approved this previous survey. In such survey, the purpose and procedures of the study were explained to all participants and written informed consent was obtained from all of them and from the next of kin of minor participants.

\section{Results}

Five $(2.5 \%)$ of the 204 patients with vision impairment and none of the 19 patients with blindness were positive for antiToxocara IgG antibodies. In total, five of the 223 (2.2\%) patients with vision impairment or blindness were positive for anti-Toxocara IgG antibodies.

None of the socio-demographic characteristics of patients including age, sex, educational level, occupational contact with soil or socio-economic status showed $\mathrm{P}$ values $<0.10$ by bivariate analysis (Table 1). With respect to the housing conditions of the patients, the variable "living in a house with soil floor" and "education of the head of the family" showed $\mathrm{P}$ values $<0.10$. Other housing variables including crowding at home, availability of drinkable water, and form of elimination of excreta showed $\mathrm{P}$ values $>0.10$. Of the clinical characteristics, seropositivity to Toxocara was not associated with the presence of underlying diseases, history of surgery, transplantation or blood transfusion, response to treatment, and presence of hearing, reflexes, or memory impairments.

Of the behavioral characteristics of patients, the variables consumption of untreated water, and eating out of home showed $\mathrm{P}$ values $<0.10$ (Table 2). Other behavioral characteristics of patients including contact with cats and dogs, foreign traveling, consumption of any type of meat, unwashed raw vegetables and fruits, and drinking unpasteurized milk showed $P$ values $>0.10$. Multivariate analysis of behavioral and hous- 
Table 1. Socio-Demographic Characteristics of the Patients Studied

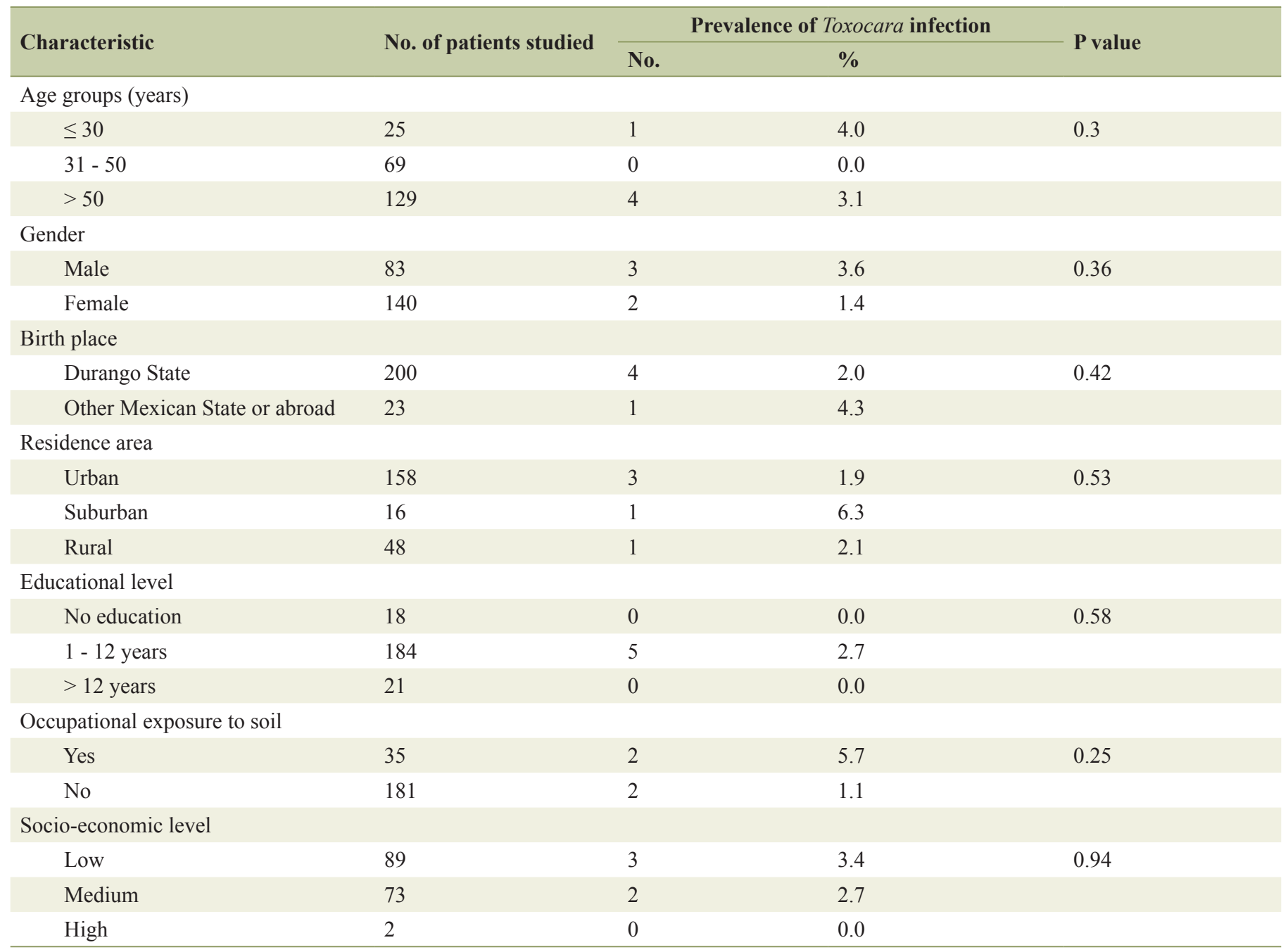

ing characteristics with $\mathrm{P}$ values $<0.10$ obtained by bivariate analysis showed that Toxocara seropositivity was associated only with the variable "living in a house with soil floor" (OR $=11.14 ; 95 \%$ CI: $1.57-78.74 ; \mathrm{P}=0.01)$. The result of the Hosmer-Lemeshow test $(\mathrm{P}=0.99)$ indicated an acceptable fit of our regression model.

\section{Discussion}

Very little is known about the seroepidemiology of Toxocara infection in Mexico. To the best of our knowledge, there is not any study about the seroepidemiology of Toxocara infection in patients suffering from vision impairment or blindness in Mexico. The present serosurvey was performed to investigate the seroprevalence and correlates of Toxocara exposure in these patients in the northern Mexican city of Durango. Results of the present study suggest a low (2.2\%) seroprevalence of Toxocara exposure in patients with blindness or vision impairment. The seroprevalence found in patients with vision impairment or blindness is lower than those (4.7-26.2\%) reported in other population groups in the region. A $26.2 \%$ seroprevalence of Toxocara exposure was found in the ethnic group of Tepehuanos in rural Durango, Mexico [17]. In a study of waste pickers in Durango City, a seroprevalence of $13 \%$ was found [18]. In addition, patients in a psychiatric hospital in the same city had a $4.7 \%$ seroprevalence of Toxocara infection [19]. However, comparison of these seroprevalences should be interpreted with care because differences in ages in populations among the studies exist. The mean age in patients with vision impairment or blindness was 50.49 years old, while mean age in Tepehuanos was 32.46 years old, in waste pickers was 36.0 years old, and in psychiatric patients was 43.57 years old. Furthermore, differences in sanitation and hygiene practices might explain the differences in Toxocara seroprevalences among the population groups. It is likely that most patients with vision impairment or blindness had had better sanitation and hygiene practices than waste pickers, rural Tepehuanos and psychiatric patients.

In the present study, none of the socio-demographic char- 
Table 2. Bivariate Analysis of Selected Behavioral and Housing Characteristics in Patients and Seroprevalence of Toxocara Infection

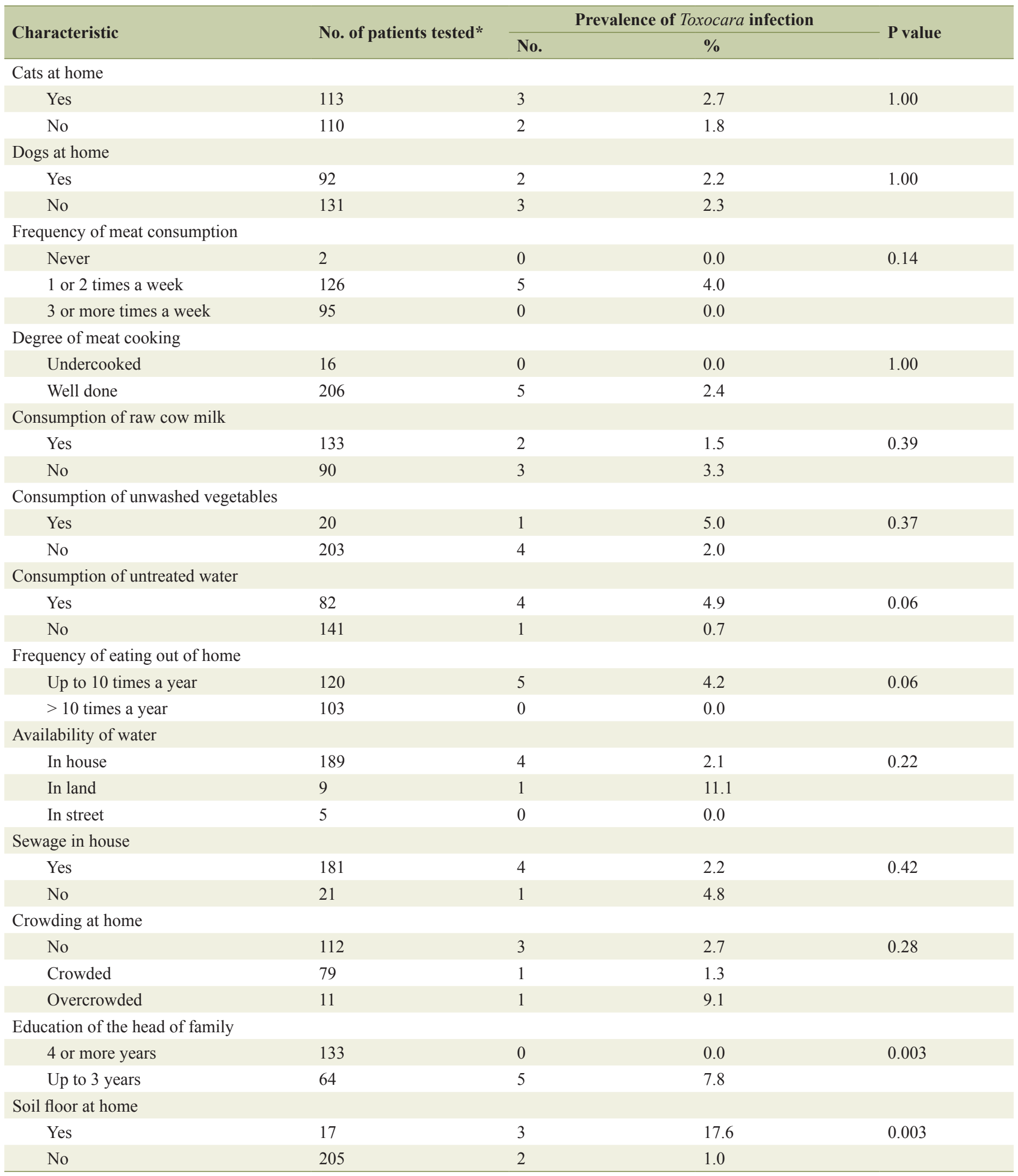

*Patients with available data. 
acteristics of the patients including age, sex, educational level, or socio-economic status was associated with Toxocara exposure. Our results are consistent with those reported in a recent study where researchers found no association of the presence of anti-Toxocara antibodies with age, gender, and socio-economic status in healthy adults in southeast Brazil [20]. Contact with cats and dogs might represent a risk for Toxocara infection; however, we did not find an association of infection and contact with cats or dogs. This result also agrees with the lack of association between these variables in the study of healthy adults in Brazil [20]. An important risk factor for Toxocara infection is contact with soil $[6,7,20]$. In our studied population, patients with occupational exposure to soil had a higher seroprevalence of Toxocara infection than those without occupational exposure to soil. However, the difference in seroprevalence among these groups was not statistically significant. It is likely that the low number of Toxocara-seropositive patients found did not allow obtaining a statistically significant difference in the seroprevalences among the groups. Interestingly, multivariate analysis showed an association of Toxocara exposure with living in a house with soil floors. To the best of our knowledge, this is first report of this association. Soil floors at home that are contaminated with excrement of Toxocara-infected cats or dogs may contribute to transmit Toxocara infection to humans. The fact that Toxocara infection was associated with soil floors at home but not with occupational exposure to soil suggests that patients had more risk to acquire Toxocara infection at home than at work. Further studies should consider searching Toxocara in soil floors at homes. In addition, people living in houses with soil floors and having cats or dogs should consider taking preventive measures against Toxocara infection, for instance, deworming of their pets. However, soil flooring at home is a reflection of poverty and people living in this disadvantaged condition cannot afford to deworm their pets. Therefore, governmental actions to aid people living in poverty for changing soil floors with concrete floors at homes and deworming of their pets are highly needed.

\section{Conclusions}

This is the first report of Toxocara exposure in patients with vision impairment and blindness in Mexico, and of an association of Toxocara exposure with living in a house with soil floors. Results suggest a low Toxocara exposure in these patients in Durango, Mexico. The risk factor associated with Toxocara exposure identified in this study deserves further investigation.

\section{Conflict of Interests}

None.

\section{References}

1. Ocular toxocariasis--United States, 2009-2010. MMWR Morb Mortal Wkly Rep. 2011;60(22):734-736.
2. Rubinsky-Elefant G, Hirata CE, Yamamoto JH, Ferreira MU. Human toxocariasis: diagnosis, worldwide seroprevalences and clinical expression of the systemic and ocular forms. Ann Trop Med Parasitol. 2010;104(1):3-23.

3. Pelloux H, Faure O. [Toxocariasis in adults]. Rev Med Interne. 2004;25(3):201-206.

4. Lee RM, Moore LB, Bottazzi ME, Hotez PJ. Toxocariasis in North America: a systematic review. PLoS Negl Trop Dis. 2014;8(8):e3116.

5. Macpherson CN. The epidemiology and public health importance of toxocariasis: a zoonosis of global importance. Int J Parasitol. 2013;43(12-13):999-1008.

6. Despommier D. Toxocariasis: clinical aspects, epidemiology, medical ecology, and molecular aspects. Clin Microbiol Rev. 2003;16(2):265-272.

7. Overgaauw PA. Aspects of Toxocara epidemiology: human toxocarosis. Crit Rev Microbiol. 1997;23(3):215231.

8. Magnaval JF, Glickman LT, Dorchies P, Morassin B. Highlights of human toxocariasis. Korean J Parasitol. 2001;39(1):1-11.

9. Dutra GF, Pinto NS, de Avila LF, Dutra PC, Telmo Pde L, Rodrigues LH, Silva AM, et al. Risk of infection by the consumption of liver of chickens inoculated with low doses of Toxocara canis eggs. Vet Parasitol. 2014;203(12):87-90.

10. Guillot J, Bouree P. [Zoonotic worms from carnivorous pets: risk assessment and prevention]. Bull Acad Natl Med. 2007;191(1):67-78; discussion 79-81.

11. Sariego I, Kanobana K, Rojas L, Speybroeck N, Polman K, Nunez FA. Toxocariasis in Cuba: a literature review. PLoS Negl Trop Dis. 2012;6(2):e1382.

12. Woodhall D, Starr MC, Montgomery SP, Jones JL, Lum F, Read RW, Moorthy RS. Ocular toxocariasis: epidemiologic, anatomic, and therapeutic variations based on a survey of ophthalmic subspecialists. Ophthalmology. 2012;119(6):1211-1217.

13. Arevalo JF, Espinoza JV, Arevalo FA. Ocular toxocariasis. J Pediatr Ophthalmol Strabismus. 2013;50(2):76-86.

14. Jimenez-Balderas FJ, Garcia-Jaimes J, Rios R, ZonanaNacach A, Tapia-Romero R, Villanueva N, Mendez-Samperio $\mathrm{P}$, et al. Isolation of $\mathrm{IgG}$ antibodies to Toxocara in ankylosing spondylitis patients with acute anterior uveitis. Korean J Ophthalmol. 2014;28(3):207-212.

15. Alvarado-Esquivel C, Liesenfeld O, Torres-Castorena A, Estrada-Martinez S, Urbina-Alvarez JD, Ramos-de la Rocha M, Marquez-Conde JA, et al. Seroepidemiology of Toxoplasma gondii infection in patients with vision and hearing impairments, cancer, HIV, or undergoing hemodialysis in Durango, Mexico. J Parasitol. 2010;96(3):505508.

16. Bronfman M, Guiscafre H, Castro V, Castro R, Gutierrez $\mathrm{G}$. [Strategies for improving the therapeutic patterns used in acute diarrhea in primary medical care units. II. The measurement of inequality: a methodologic strategy, analysis of the socioeconomic features of the sample]. Arch Invest Med (Mex). 1988;19(4):351-360.

17. Alvarado-Esquivel C. Seroepidemiology of toxocariasis 
in a rural Tepehuanos population from Durango, Mexico. J Helminthol. 2014;88(2):173-176.

18. Alvarado-Esquivel C. Toxocariasis in waste pickers: a case control seroprevalence study. PLoS One. 2013;8(1):e54897.

19. Alvarado-Esquivel C. Toxocara infection in psychiatric inpatients: a case control seroprevalence study. PLoS One. 2013;8(4):e62606.

20. Negri EC, Santarem VA, Rubinsky-Elefant G, Giuffrida R. Anti-Toxocara spp. antibodies in an adult healthy population: serosurvey and risk factors in Southeast Brazil. Asian Pac J Trop Biomed. 2013;3(3):211-216. 